\title{
Lung Tumor Segmentation and Staging from CT Images Using Fast and Robust Fuzzy C-Means Clustering
}

\author{
Rupak Bhakta \\ Department of Electronics and Communication Engg., \\ Khulna University of Engineering \& Technology, Bangladesh \\ Email: rupakbhakta123@gmail.com \\ A. B. M. Aowlad Hossain \\ Department of Electronics and Communication Engg., \\ Khulna University of Engineering \& Technology, Bangladesh \\ Email: aowlad0403@ece.kuet.ac.bd
}

Received: 08 August 2019; Accepted: 24 October 2019; Published: 08 February 2020

\begin{abstract}
Lung tumor is the result of abnormal and uncontrolled cell division and growth in lung region. Earlier detection and staging of lung tumor is of great importance to increase the survival rate of the suffered patients. In this paper, a fast and robust Fuzzy c-means clustering method is used for segmenting the tumor region from lung $\mathrm{CT}$ images. Morphological reconstruction process is performed prior to Fuzzy cmeans clustering to achieve robustness against noises. The computational efficiency is improved through median filtering of membership partition. Tumor masks are then reconstructed using surface based and shape based filtering. Different features are extracted from the segmented tumor region including maximum diameter and the tumor stage is determined according to the tumor staging system of American Joint Commission on Cancer. 3D shape of the segmented tumor is reconstructed from series of 2D CT slices for volume measurement. The accuracy of the proposed system is found as $92.72 \%$ for 55 randomly selected images from the RIDER Lung CT dataset of Cancer imaging archive. Lower complexity in terms of iterations and connected components as well as better noise robustness are found in comparison with conventional Fuzzy c-means and k-means clustering techniques.
\end{abstract}

Index Terms-Fuzzy c-means clustering, morphological reconstruction, noise robustness, computational efficiency, lung tumor segmentation, tumor staging

\section{INTRODUCTION}

Lung tumor is the disease of uncontrolled cell division and growth of lung tissues that causes severe health risks. Lung tumor might be cancerous or noncancerous. Smoking, pollution of air, use of harmful chemicals etc. are the main reasons behind lung cancer. Only one side of the lung and nearby lymph nodes is affected by cancer at the first stage. It can grow from there into both the lungs and the areas around the lungs, or into distant organs. Since lung cancer in the earlier stages does not show apparent symptoms, diagnosis often confirms after it has spread. Among all cancer types, lung cancer survival rate is the lowest after diagnosis. It is believed that the rate of survival may rise if the lung tumor can be detected and staged early [1]. Stages of cancer reflect how far the cancer has spread and staging can help for possible treatment or therapeutic prescription.

Computed tomography (CT) image is one of the most popular means for the detection of lung tumors as they provide anatomical and pathological information on various sections of the lung [2]. For precise treatment, accurate segmentation and staging of lung tumors from lung CT images is of great importance. The 3D reconstruction of tumor using segmented $2 \mathrm{D}$ tumors from series of CT slices can improve the staging accuracy providing more accurate volumetric information.

Modern applications of medical image processing include numerous useful segmentation techniques for lung tumor detection and classification. The accuracy of lung tumor detection and staging mostly depends on the accuracy of the applied technique of segmentation. In addition, the computational complexity of the segmentation techniques determines the time needed for the segmentation process. It is therefore desirable to use a segmentation method with high precision and less computational complexity for lung tumor detection.

Many different works have been done and reported in literature previously to detect and classify the lung tumor from CT image using various types of algorithms [3-14]. Lung tumor is often segmented manually which is user and experience dependent, subjective, and may lead to erroneous diagnosis. Thresholding is the simplest type of image segmentation technique[5], which is very fast but 
not so efficient. Algorithms based on machine learning like support vector machine (SVM) [6], artificial neural network (ANN) [7,8] etc. can provide satisfactory performance for segmentation and classification tasks with supported preprocessing steps, but training data is required by these algorithms. The performance of these algorithms declines without enough training data. Recently deep neural networks are becoming popular to address classification problems $[9,10]$. But large training datasets and huge computational cost are needed for deep learning. Different clustering formulas can be used for tumor segmentation as these are fairly accurate and does not require any types of training data. K-means clustering [11] is the simplest type of hard clustering algorithm but it is susceptible to noise and so without proper pre-processing method cannot segment the targeted regions accurately. Fuzzy c-means (FCM) [12] is better than other hard clustering algorithms because it has more ambiguity tolerance and preserves more original picture data. However, it fails to properly segment images with complicated texture and background because it only acknowledges gray level intensity but does not consider the spatial information. For the same reason, it cannot segment noise-corrupted images accurately. To overcome the drawbacks of k-means clustering and FCM, various enhanced clustering algorithms have been used to segment lung tumor like Gustafson-Kessel clustering [13] EK-Mean clustering [14] etc. However these algorithms are also more or less susceptible to noise corruption. So for accurate segmentation, noise filtering is essential as preprocessing step where choosing the filter is a cumbersome task without knowing the noise type in advance.

The requirement of a faster and accurate segmentation algorithm is still a demanding and challenging task for efficient detection and staging of lung tumor. Therefore, in this paper, we have planned to use a fast and robust Fuzzy c-means clustering method (FRFCM) [15] to segment tumor from lung CT images with strategic viewpoints of achieving robustness against noise and faster but accurate segmentation performance. FRFCM is an enhanced FCM algorithm which depends on morphological reconstruction and membership filtering that is significantly faster and more robust than FCM. It is effective for the segmentation of images corrupted by noise because the isolated pixels are removed by reconstruction process and the filtering of memberships can effectively improve the matrix of membership partitions. After segmenting the CT image a surface based and a shape based filter is used to detect tumor from the segmented image. Then using the maximum diameter of the detected tumor, different sub-stages will be determined according to the Tumor, Lymph nodes, and Metastasis (TNM) classification criteria [16]. Segmented tumor regions from a set of CT slices are used to reconstruct the $3 \mathrm{D}$ shape in order to determine the volume of the tumor.

The rest of the paper is organized as follows: the next section describes the proposed methodology which describes the segmentation task using FRFCM technique with related post processing, feature extractions and classification strategy. Third section presents the description of data used in this study as well as analysis, comparison and discussion on obtained results which are followed by the conclusion section.

\section{Methodology}

In this proposed method, first the lung CT slices is segmented using FRFCM algorithm. As morphological reconstruction is performed on the image as a part of FRFCM, no other pre-processing of the image like noise filtering is required in this process. Fig. 1 shows the step by step process of the proposed lung tumor segmentation and staging methodology. The explanation of Fig. 1 is given in details in the flowing subsections titled FRFCM based Segmentation and Feature Extraction and Tumor Staging.

\section{A. FRFCM based Segmentation}

In this process, the optimization of the data distribution is achieved by morphological reconstruction. After that FCM clustering algorithm is applied on the histogram of morphologically reconstructed image. Finally modification of membership partition is achieved using a median filter.

We have used lung CTs from the RIDER dataset available in Cancer Imaging Archive [17]-[19]. In some cases, where the lung tumor is connected with other tissue, its region cannot be segmented properly by taking the entire CT image [20] as the tumor is segmented with similar other tissues. In such cases, we preliminarily separate the lung tumor from wall or other tissue regions through selecting arbitrary shape of the tumor. Then the FRFCM segmentation process is applied only on the selected region.

\section{Morphological Reconstruction}

FCM is not efficient in case of noisy images as noise always affects the distribution features of the data, which may create two issues [15]. Firstly the output acquired by the FCM is inefficient and another issue is that FCM needs greater number of iterations for a noisy image. To solve these issues, morphological reconstruction process is used in FRFCM before the core FCM algorithm to remove corrupted noise.

Morphology describes the structure and shape of the component in an image in the image processing context. Morphological operations operate on the simple basis of set theory and depend on pixel relative order rather than the numerical values [21]. Typical morphological operations only require one image and one structuring element. However, reconstruction is a kind of morphological transformation requiring two images and a single structuring element. One image functions as the beginning point for the reconstruction process and is known as marker image. The function of the other image is to control the transformation and is known as the mask. The function of the structuring element is to define connectivity. 


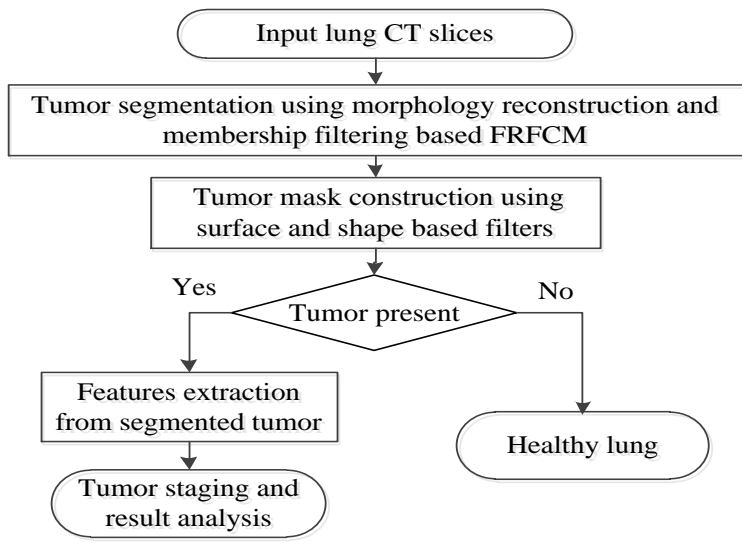

Fig. 1. Flowchart of proposed methodology

If $G$ is considered as the mask image and $F$ is considered as the marker image, the morphological reconstruction of $G$ from $F$, denoted as $R_{G}(F)$, is described by the following iterative procedure:

1. $h_{1}$ is initialized to be the marker image, $F$.

2. Structuring element $B$ matrix is created.

3. Repeat: $h_{(k+1)}=\left(h_{k} \otimes B\right) \cap G$, until $h_{(k+1)}=h_{k}$

$$
R_{G}(F)=h_{(k+1)}
$$

Marker image $F$ is a subset of $G: F \subseteq G$. The $3 \times 3$ matrix $B$ with all elements one is used as structuring element.

Morphological reconstruction can optimize the distribution of information without taking the noise type into account. So, morphological reconstruction eliminates the trouble of selecting filters for noise corrupted images. It implements spatial data into FCM to obtain a more improved segmentation result.

\section{Membership Filtering}

The enhanced FCM algorithms are less sensitive to noises and show better overall performance for segmentation by adding local spatial information to the objective function of the FCM algorithm. The computational complexity of these algorithms is controlled by the fuzzy factor of the objective function. The fuzzy factor regulates the effect of neighborhood pixels on the central pixel. In FRFCM, the contribution of the fuzzy factor is replaced by the spatial neighborhood information of membership partition. To improve segmentation results, the inclusion of local spatial data is comparable to membership filtering. Membership filtering, however, does not need the distances between pixels of the clustering centers and the local spatial neighbors to be calculated. The correlating computational complexity of FRFCM algorithms premised on membership filtering is therefore less than other existing algorithms.

To modify the membership partition, a median filter is used. Median filter itself is a simple type of filter, but if it is implemented on the partition matrix after each iteration, the algorithm will become more complex and less time- efficient. So the filtering is only used on the endmost membership partition matrix which can be found after finishing all iterations. Also if the window size is too large, it results in better filtering for images with high noise density, but the filtering needs increased computations. So for optimal computational efficiency, a small $3 \times 3$ filtering moving window $(w)$ is used in this study for median filtering.

\section{Algorithm for FRFCM Segmentation}

1. First the number of cluster $(c)$, the Fuzzification parameter $(m)$, the filtering window size $(w)$ and the loop minimum error threshold $(\eta)$ is set.

2. New image $F$ is computed using morphological reconstruction,

$$
F=R^{c}(f)
$$

Where $R^{c}$ denotes morphological closing reconstruction, $f$ represents an original image and $F_{l}$ is a gray level, $1 \leqslant l \leqslant q, q$ denotes the number of the gray levels contained in $F$.

3. Then the histogram of the new image is computed.

4. The membership partition matrix is randomly initialized $U^{(0)}$

5. The loop counter is set as $t=0$.

6 . The clustering centers are updated using:

$$
v_{k}=\frac{\sum_{l=1}^{q} \gamma_{l} u_{k l}^{m} F_{l}}{\sum_{l=1}^{q} \gamma_{l} u_{k l}^{m}}
$$

Where $v_{k}$ is the center of cluster $k$, fuzzy membership of gray value $l$ with respect to cluster $k$ is represented by $u_{k l}$, and $\sum_{l=1}^{q} \gamma_{l}=N$. Here, $\gamma_{l}$ is the number of pixels in gray level $F_{l}$ and $N$ is the total number of pixels in the image.

7. The membership partition matrix $U^{(t+1)}$ is updated using:

$$
u_{k l}=\frac{\left\|F_{l}-v_{k}\right\|^{-\frac{2}{m-1}}}{\sum_{j=1}^{c}\left\|F_{l}-v_{k}\right\|^{-\frac{2}{m-1}}}
$$

8. If $\max \left\{U^{(t)}-U^{(t-1)}\right\}<\eta$ then stop, otherwise, set $t=$ $t+1$ and go to Step 6.

9. Median filtering is implemented on membership partition matrix $U^{\prime}$ : 


$$
U^{\prime \prime}=\operatorname{med}\left\{U^{\prime}\right\}
$$

\section{Surface and Shape based Filtering}

Automatic detection process is easier if the tumor is not connected with other tissues. A binary version of the segmented image is created. All the connected components (objects) in the binary image are discovered. The accuracy of the detection depends on:

1. The surface and shape of the components,

2. The connectivity parameter,

3. Whether objects are touching or not.

Surface based filter and shape based filter are used to remove other objects except only the tumor region.

Surface base filtering used on the segmented image to remove too large surfaced regions like the lung wall or small surfaced regions like lung vessels. The surface of each different component of the segmented image is measured by counting total number of pixels of each component. The surface $(S)$ of a component is determined according to the formula:

$$
S=n_{p} s_{p}
$$

Where $n_{p}$ is number of pixels in that particular component and $s_{p}$ is pixel spacing. Two threshold surface values are set for the filter. Large surfaced lung wall gets removed as it has bigger surface than the higher threshold value. The isolated pixels or too small surfaced than suspected tumor are also eliminated having smaller surface area than the lower threshold.

After filtering the components by a surface based filter, the remaining components are introduced to a filter which filters the components based on their shape. The perimeter of each component is measured and then the roundness $(R)$ of each observed components is determined according to the formula:

$$
R=\frac{4 \pi S}{L^{2}}
$$

Where, area of the component is represented by $S$ and the perimeter is represented by $L$. When the value of $R$ for a component is close to 1 , then the component shape is close to the shape of a circle. Using the value of $R$, some too oddly shaped components to be the tumor but having the surfaces in the surface range of a small tumor is removed from the segmented image.

\section{B. Feature Extraction and Tumor Staging}

In practice, medical imaging consist metadata which provide various information along with the image, such as width, height, bit depth, pixel spacing etc. These metadata are helpful to determine various features of the tumor from the segmented image. Tumor surface area, maximum diameter and centroid position are chosen as potential features for tumor staging which can be calculated form the pixel information of the segmented tumors and the metadata on acquisition systems and settings. From the segmented tumor, the total pixels in the tumor region were identified and used to determine various features. Multiplying the total number of pixels in the tumor region with the pixel spacing, the surface area of the tumor in the CT image can be found. The centroid position of the tumor is also determined to learn the relative location of the tumor. The average diameter of the tumor is determined by measuring the diameter of same surfaced circle. For calculating the maximum diameter of the tumor, the distances between each two pixels of the edge of the tumor is measured. Maximum diameter of tumor is the main criteria of $\mathrm{T}$ staging. The segmented tumor regions from a set of CT slices are used to reconstruct the $3 \mathrm{D}$ shape in order to determine the volume of the tumor.

TNM system is used to classify lung tumor into basic stages based on different criteria. Here, the characteristics of the primary tumor are represented by $\mathrm{T}$ sub-stages, nodal participation is represented by $\mathrm{N}$ sub-stages and distant metastasis is represented by $M$ sub-stages. We have classified tumors into various $\mathrm{T}$ sub-stages according to TNM staging rules [16] and considering the main criterion is the maximum diameter as shown in Table 1 . We did not classify the tumor into $\mathrm{N}$ and $\mathrm{M}$ substages, because information of regional and distant metastasis is needed for that.

\section{Result ANALYSIS AND Discussions}

The RIDER Lung CT dataset from the website of Cancer imaging archive is used in this work. The dataset includes 32 patients suffering from non-small cell lung cancer, everyone of whom was using the same kind of imaging procedure and underwent two chest CT scans within fifteen minutes. There are 63 series and a total of 15,419 CT images in this dataset [17-19]. The lung CT images are of Digital Imaging and Communications in Medicine (DICOM) [22] format metadata. The proposed segmentation process and the subsequent feature extraction and staging steps have been performed through programming in MATLAB R2016a (The MathWorks Inc., Massachusetts, USA). As the slice based 2D segmentation is major focus of this study, the 3D reconstruction of the tumor region was executed in $3 \mathrm{D}$ Slicer software platform [23].

\section{A. Tumor Segmentation and Staging Results}

Fig. 2 shows five different cases of lung CT image at different processing steps under FRFCM based segmentation. The original CT image of different cases is shown in first column and the resultant images after morphological reconstruction are in second column of Fig. 2 respectively. In this case, morphological closing reconstruction was performed. The third column of Fig. 2 shows the resultant FRFCM segmented CT images. After the surface and shape based filtering, the detected tumor is shown in the last column of Fig. 2. 
Table 1. Sub-Stages and their corresponding Maximum Diameters [16]

\begin{tabular}{|c|c|}
\hline Sub-Stage (T) & Maximum Diameter \\
\hline TX & Not Assessed \\
\hline T0 & No tumor \\
\hline T1a & $<=1 \mathrm{~cm}$ \\
\hline T2b & $>1 \mathrm{~cm}$ and $<=2 \mathrm{~cm}$ \\
\hline T1c & $>2 \mathrm{~cm}$ and $<=3 \mathrm{~cm}$ \\
\hline T2a & $>3 \mathrm{~cm}$ and $<=4 \mathrm{~cm}$ \\
\hline T2b & $>4 \mathrm{~cm}$ and $<=5 \mathrm{~cm}$ \\
\hline T3 & $>5 \mathrm{~cm}$ and $<=7 \mathrm{~cm}$ \\
\hline T4 & $>7 \mathrm{~cm}$ \\
\hline
\end{tabular}

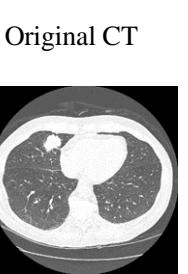

$\mathrm{a}(1)$

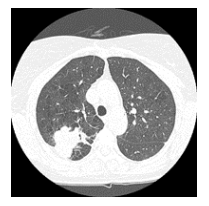

$\mathrm{a}(2)$

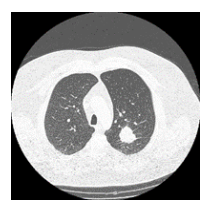

$\mathrm{a}(3)$

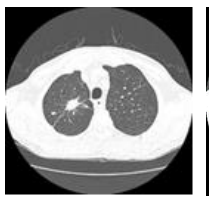

$\mathrm{a}(4)$

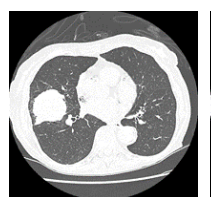

$\mathrm{a}(5)$
Morphological
Reconstructed

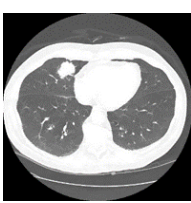

$b(1)$

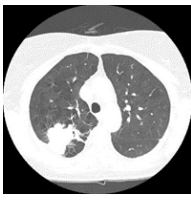

$b(2)$

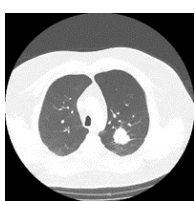

$b(3)$

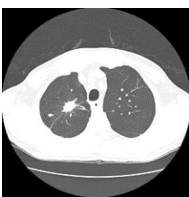

$b(4)$

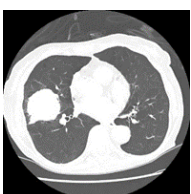

$b(5)$

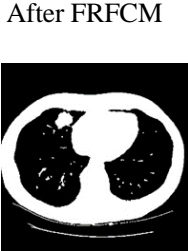

$\mathrm{c}(1)$

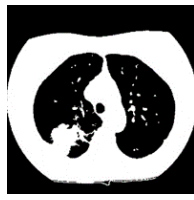

$c(2)$

c(3)

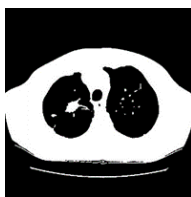

c(4)

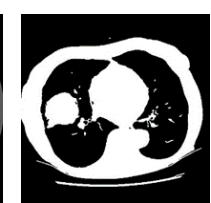

$c(5)$

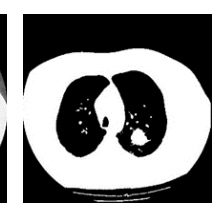

Segmented Tumor

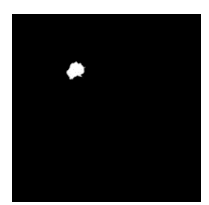

$\mathrm{d}(1)$

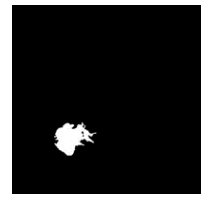

$d(2)$

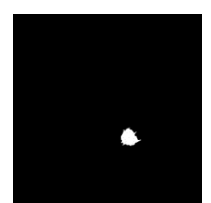

$\mathrm{d}(3)$

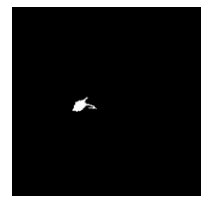

d(4)

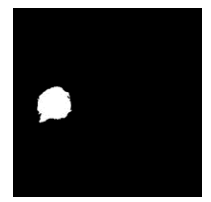

$\mathrm{d}(5)$

Fig. 2. Stepwise results of tumor segmentation for five different cases

Surface area, maximum diameter, and centroid position features are calculated using pixel information of the segmented tumors and the metadata available in RIDER datasets. The surface area and the centroid positions are useful to locate the position and estimating volume of the tumor. The staging of the tumors are then performed

following maximum diameter conditions shown in the Table 1. The calculated features and staging of 5 tumor cases of Fig. 2 are shown in Table 2.

Table 2. Extracted Features and Staging of Different Tumor Cases

\begin{tabular}{|c|c|c|c|c|}
\hline $\begin{array}{c}\text { Case } \\
\text { No. }\end{array}$ & $\begin{array}{c}\text { Surface } \\
\left(\mathrm{mm}^{2}\right)\end{array}$ & $\begin{array}{c}\text { Centroid } \\
\text { position }\end{array}$ & $\begin{array}{c}\text { Maximum } \\
\text { Diameter }(\mathrm{mm})\end{array}$ & Stage \\
\hline 1 & 799.791 & $\begin{array}{r}{[171.863,} \\
155.592]\end{array}$ & 38.3355 & $\mathrm{~T} 2 \mathrm{a}$ \\
\hline 2 & 1741.9 & $\begin{array}{r}{[161.668,} \\
359.566]\end{array}$ & 65.4152 & $\mathrm{~T} 3$ \\
\hline 3 & 922.541 & $\begin{array}{r}{[314.558,} \\
335.294]\end{array}$ & 42.2066 & $\mathrm{~T} 2 \mathrm{~b}$ \\
\hline 4 & 474.052 & $\begin{array}{r}{[189.969,} \\
266.830]\end{array}$ & 43.9537 & $\mathrm{~T} 2 \mathrm{~b}$ \\
\hline 5 & 2256.3 & $\begin{array}{r}{[111.333,} \\
261.249]\end{array}$ & 65.4679 & $\mathrm{~T} 3$ \\
\hline
\end{tabular}

\section{B. Performance Evaluation}

A higher number of true positive $(T P)$ and true negative $(T N)$ as well as a lower number of false positives $(F P)$ and false negatives $(F N)$ are desired in the detection process. False positives occur when there is no tumor but the system determines that it exists and false negatives occur when there is tumor but the system determines that it does not exist. Whereas, true positive and true negative reflects the actual presence or absence of the target. The performance of the method is evaluated in terms of the three parameters i.e., sensitivity $(S E)$, specificity $(S P)$ and accuracy.

Sensitivity measures the ability of appropriately detected positive cases and defined as:

$$
S E=\frac{T P}{T P+F N}
$$

Specificity measures the ability of appropriately detected negative cases and expressed as:

$$
S P=\frac{T N}{F P+T N}
$$

Accuracy measures that the proportion of correct classified events and defined as:

$$
\text { Accuracy }=\frac{T P+T N}{T P+F P+T N+F N}
$$

For measuring sensitivity, specificity and accuracy of the detection system, randomly 25 healthy lung CT images and 30 lung CT images with non-small cell lung cancer was taken. Among the total 55 cases, the number of tumor correctly identified is $28(T P)$ and not identified is $2(F N)$, and number of healthy cases correctly identified is $23(\mathrm{TN})$ and falsely identified as affected is $2(F P)$. These data are prepared from the comparison between segmented output and the tumor availability information given in the original database. Then the sensitivity, specificity and accuracy are obtained as $92 \%, 93.33 \%$, and $92.72 \%$ respectively using (8) to (10). 


\section{Performance Investigation against Noise}

As robustness against noise is a major concern of this work and FRFCM is supposed to be more robust against noise than other algorithms, we have investigated the performance of the proposed method against different levels of noise corrupted lung CT images. In order to do that firstly, the tumor detected from the noiseless CT image is considered as the ground truth reference. Then we have added salt and pepper noise with various noise densities to the CT image and determined the similarity of detected tumor with the ground truth reference. The similarity is calculated using 2D correlation coefficient. Table 3 shows the performance of the proposed method against corrupted images with different levels of noise densities. Here, noise density represents the percentage of total pixels in the image which will be affected by the added noise. So, a noise density of $10 \%$ means $10 \%$ of total pixels of the image is corrupted. In Table 3 , it is seen that, the tumors detected with different noise density are quite accurate up to $10 \%$ noise level. In spite slight decline after $10 \%$, the proposed method performs satisfactorily up to $40 \%$ noise level.

Table 3. Performance against Various Noise Densities

\begin{tabular}{|c|c|c|c|}
\hline \multicolumn{2}{|c|}{ Case - 1 } & \multicolumn{2}{c|}{ Case - 2 } \\
\hline $\begin{array}{c}\text { Noise } \\
\text { density (\%) }\end{array}$ & $\begin{array}{c}\text { Similarity with } \\
\text { reference (\%) }\end{array}$ & $\begin{array}{c}\text { Noise } \\
\text { density (\%) }\end{array}$ & $\begin{array}{c}\text { Similarity with } \\
\text { reference (\%) }\end{array}$ \\
\hline 2 & 99.97 & 2 & 99.94 \\
\hline 5 & 99.16 & 5 & 99.52 \\
\hline 10 & 99.70 & 10 & 99.36 \\
\hline 20 & 97.68 & 20 & 97.18 \\
\hline 40 & 96.02 & 40 & 96.25 \\
\hline
\end{tabular}

According to the data shown in the Table 3 for both two different lung CT images cases, the robustness of proposed FRFCM against noises is clearly noticeable. The noise robustness of FCM and k-means clustering algorithm has also been investigated considering similar noisy images as used in FRFCM case in Table 3. On average, for FCM and k-means algorithm, the detected tumor from a noisy image with below $10 \%$ noise density is about $90 \%$ similar with the noiseless reference tumor. But these algorithms fail to detect in case of over $10 \%$ noisy CT images. It can be seen in Table 3 that the detected tumor with FRFCM algorithm from a noisy image with $10 \%$ noise density is over $99 \%$ similar. Even at $40 \%$ noise density, the tumors detected with FRFCM algorithm are above $96 \%$ similar with the ground truth reference tumor. Over $50 \%$ noise density, it was found that the FRFCM algorithm also fails to detect the tumor.

\section{Comparison of Comutational Complexity with other Clustering Algorithms}

The execution time of the method is depends on computational complexity which is important for faster operation considering real time diagnosis. The reduced number of remaining connected components after segmentation lowers the computational complexity for tumor mask preparation. To compare their performance by measuring the connected components in the images, we have segmented the same lung CT images with FRFCM, FCM and k-means clustering algorithm using MATLAB programming as shown in Fig. 3. It can be seen that FRFCM gives significantly lower number of connected components than FCM and k-means. That means it is better at removing isolated pixels and so can produce a better segmentation result as well as less time is required for tumor mask extraction.

The computational complexity also depends on the number of iteration required for execution of FCM or FRFCM. Fig. 4 shows the number of iterations for FCM or FRFCM to segment 8 different cases of lung CT images from RIDER Lung CT dataset. It can be seen that FRFCM require mainly less or sometimes same number of iterations than FCM for segmentation. Therefore, FRFCM has the ability of achieving faster segmentation performance than FCM with less number of iterations.

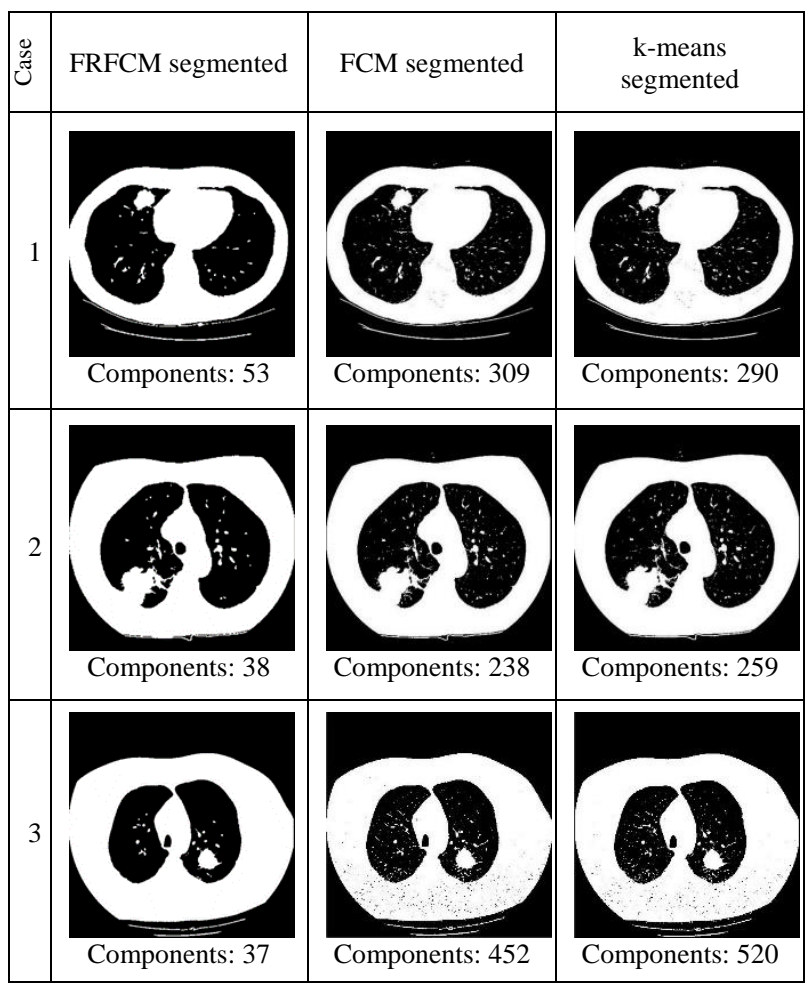

Fig. 3. Comparison of FRFCM segmentation results with other clustering algorithms in terms of connected components

We have also computed the execution time for different clustering algorithm considering tumor segmentation for different CT images using MATLAB in an Intel Core(TM) i7-8550U@1.80GHz on 64 bit Windows 10 operating system with 8GB RAM. Fig. 5 shows a comparative plot of the times that required for tumor detection by using FRFCM, FCM and k-means clustering algorithm for 10 arbitrary CT images of RIDER Lung CT dataset. From this comparison graph, it can be clearly observed that FRFCM needs much less 
execution time than FCM and k-means clustering algorithm. Furthermore, it is also noticeable that the FRFCM requires almost similar execution time for different CT image cases whereas for FCM and k-means clustering algorithm the required times are quite inconsistent.

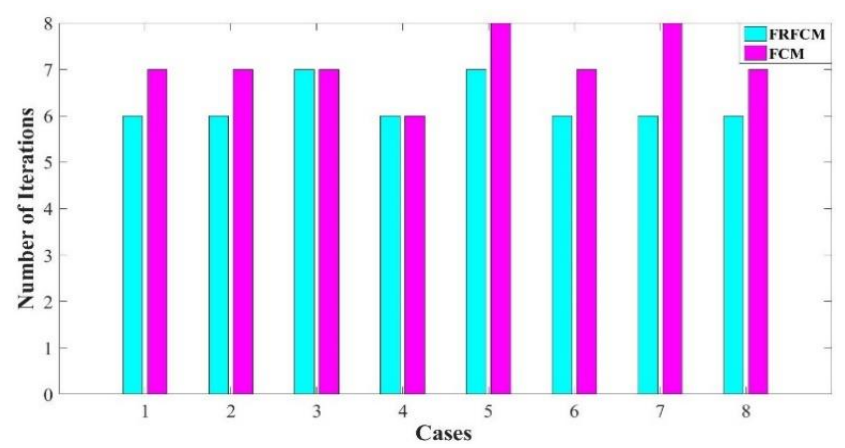

Fig. 4. Comparison of number of iterations for FCM and FRFCM

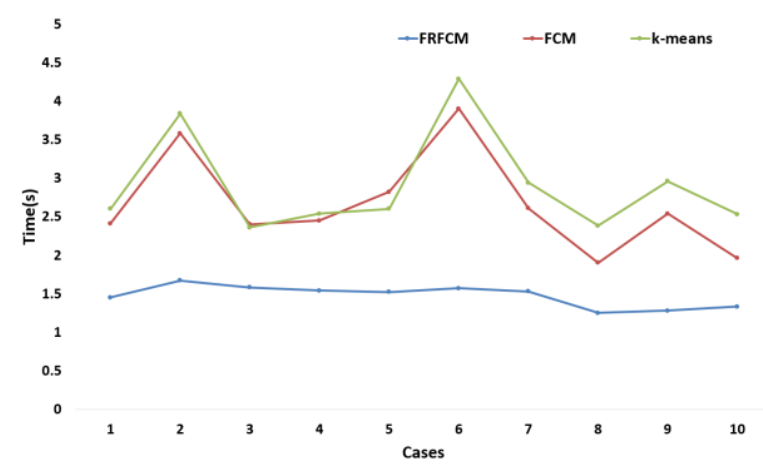

Fig. 5. Comparison of computational time for tumor detection using FRFCM, FCM, and k-means clustering algorithms

\section{E. 3D Reconstruction and Volume Measurement}

Table 4. 3D Reconstruction and Volume Measurement of Tumor

\begin{tabular}{|c|c|c|}
\hline Case No. & 3D Tumor & Volume $\left(\mathbf{c m}^{\mathbf{3}}\right)$ \\
\hline & & \\
\hline & & \\
& & \\
& & \\
& & \\
& & \\
& & \\
\hline
\end{tabular}

Though slice based 2D segmentation is major focus of this study, 3D volumetric reconstruction of the tumor is also done as volumetric information has importance for treatment or surgery. A series of lung CT slices are required for $3 \mathrm{D}$ reconstruction and volume measurement of the tumor. The thickness of CT slice is given in the metadata. After segmenting the lung tumor and constructing the tumor mask using the proposed method, the slice by slice separated regions of interest are used to reconstruct the whole tumor using 3D Slicer software platform [23]. For improvement of smoothness, thresholding is used on the 3 -D region. Then the volume of the reconstructed tumor can be measured using the pixel spacing obtained from metadata. The reconstructed tumor and their volumes are shown in Table 4 for two different tumor cases.

\section{CONCLUSION}

In this paper, a fast and robust Fuzzy c-means clustering algorithm is used for segmentation of lung tumor from the CT due to its accuracy considering robustness against noise and less computational complexity. The tumor region mask was constructed from the segmented image by using surface and shape based filtering. The centroid and size of the tumor are the determined, and these features are extracted for tumor staging according to well accepted TNM classification rules [16]. Segmentation of the tumor regions from a set of slices of CT image also leads the way of 3D reconstruction and volume estimation of the tumor. The accuracy of the proposed morphological reconstruction and membership filtering based FRFCM method has been found as $92.72 \%$ for randomly chosen 55 lung CTs. Moreover the robustness of the FRFCM technique has been tested against different percentage levels of noise corrupted images of raw CT and found noticeable similarity of detected tumor. Furthermore, it is found that the computational complexity and time of FRFCM are significantly less and consistent comparing with FCM and k-means clustering methods. However, achieving more accuracy using the FRFCM is future prospect of this work. The findings of this work are expected to be useful for clinical applications of accurate and faster segmentation and staging of lung tumor from CT.

\section{ACKNOWLEDGMENT}

The authors would like to thank the authority of Khulna University of Engineering \& Technology for laboratory support and The Cancer Imaging Archive for RIDER Lung CT data.

\section{REFERENCES}

[1] C. Zappa and S. A. Mousa, "Non-small cell lung cancer: current treatment and future advances," Transl Lung Cancer Res., vol. 5 no. 3, pp. 288-300, June 2016.

[2] W. R. Webb, "High resolution lung computed tomography. Normal anatomic and pathologic findings." Radiol Clin North Am., vo. 29, no. 5, pp. 1051-1063, September 1991.

[3] V. Ginneken, B. M. ter Haar Romeny, and M. Viergever. "Computer-aided diagnosis in chest radiography: A survey," IEEE Trans. Med. Imag., vol. 20, pp.1228-1241, 2001.

[4] N. Panpaliya, N. Tadas, S. Bobade, R. Aglawe, and A. Gudadhe, "A survey on early detection and prediction of lung cancer," International Journal of Computer Science and Mobile Computing, vol. 4, no. 1, pp. 175-184, 2015.

[5] S. Uzelaltinbulat and B. Ugur, "Lung tumor segmentation algorithm," in 9th International Conference on Theory and 
Application of Soft Computing, Computing with Words and Perception, 22-23 August 2017.

[6] G. Xiuhua et al., "Support vector machine prediction model of early-stage lung cancer based on curvelet transform to extract texture features of CT Image," International Journal of Biomedical and Biological Engineering, vol. 4, no. 11, pp. 539-543, 2010.

[7] R. N. G. Naguib and G. V. Sherbet, Artificial Neural Networks in Cancer Diagnosis, Prognosis, and Patient Management, CRC Press, 2001.

[8] E. Dandil et al., "Artificial neural network-based classification system for lung nodules on computed tomography scans," in 6th International Conference of Soft Computing and Pattern Recognition, pp.382-386, 2014.

[9] G. Jakimovski, D. Davcev, "Using double convolution neural network for lung cancer stage detection," Appl. Sci., vol. 9, no. 3, pp. 427, 2019.

[10] C. Zhang et al., "Toward an expert level of lung cancer detection and classification using a deep convolutional neural network," The Oncologist, April 2019.

[11] P. Sarker, M. M. H. Shuvo, Z. Hossain, and S. Hasan, "Segmentation and classification of lung tumor from 3D CT image using k-means clustering algorithm," in 4th International Conference on Advances in Electrical Engineering, 2017.

[12] S. Sivakumar and C. Chandrasekar, "Lung Nodule Segmentation through Unsupervised Clustering Models," Procedia Engineering, vol. 38, pp. 3064-3073, 2012.

[13] P. Afshar, A. Ahmadi, and M. H. F. Zarandi, "Lung tumor area recognition in CT images based on Gustafson-Kessel clustering," in IEEE International Conference on Fuzzy Systems, 2016.

[14] P. B. Sangamithraa and S. Govindaraju, "Lung tumour detection and classification using EK-Mean clustering," in International Conference on Wireless Communications, Signal Processing and Networking, 2016.

[15] T. Lei et al., "Significantly fast and robust fuzzy c-means clustering algorithm based on morphological reconstruction and membership filtering," IEEE Transactions on Fuzzy Systems, vol. 26, pp. 3027-3041, 2018.

[16] F. C. Detterbeck, "The eighth edition TNM stage classification for lung cancer: What does it mean on main street?," The Journal of Thoracic and Cardiovascular Surgery, vol. 155, pp. 356-359, 2018.

[17] B. Zhao, L. H. Schwartz, and M. G. Kris, "Data from RIDER Lung CT," The Cancer Imaging Archive, 2015.

[18] B. Zhao et al., "Evaluating variability in tumor measurements from same-day repeat CT scans of patients with non--small cell lung cancer," Radiology, vol. 252, pp. 263-272, 2009.

[19] K. Clark et al., "The Cancer Imaging Archive (TCIA): maintaining and operating a public information repository," Journal of Digital Imaging, vol. 26, pp. 1045$1057,2013$.
[20] Y. Nian et al., "Graph-based unsupervised segmentation for lung tumor CT images," in 3rd IEEE International Conference on Computer and Communications, 2017.

[21] R. C. Gonzalez and R. E. Woods, Digital Image Processing, Fourth edition, Pearson, 2018.

[22] B. Liu, M. Zhu, Z. Zhang, C. Yin, Z. Liu and J. Gu, "Medical image conversion with DICOM," in Canadian Conference on Electrical and Computer Engineering, 2007.

[23] A. Fedorov et al., "3D Slicer as an Image Computing Platform for the Quantitative Imaging Network," Magnetic Resonance Imaging, vol. 30, pp. 1323-1341, 2012.

\section{Authors' Profiles}

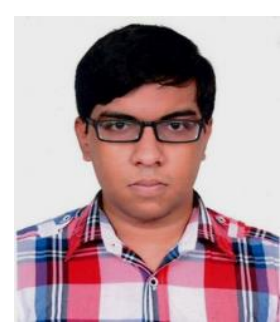

Rupak Bhakta graduated from Department of Electronics and Communication Engineering of Khulna University of Engineering \& Technology, Bangladesh in 2019 with second position in his batch. His research interests are artificial intelligence, machine learning applications, medical image processing, and computer aided diagnosis etc.

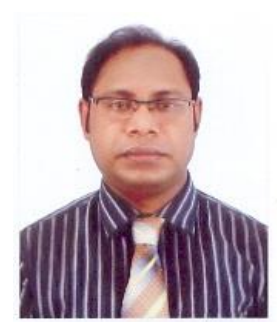

Prof. Dr. A. B. M. Aowlad Hossain is a Professor of the Department of Electronics and Communication Engineering in Khulna University of Engineering \& Technology, Bangladesh. He completed his $\mathrm{PhD}$ in Biomedical Engineering form Kyung Hee University, Republic of Korea in 2012. He obtained his M.Sc. in Electrical and Electronic Engineering Degree from Bangladesh University of Engineering \& Technology in 2005 and B.Sc. in Electrical and Electronic Engineering Degree from Khulna University of Engineering \& Technology (KUET) in 2002. He is an active researcher in the fields of Biomedical Signal and Image Processing, Elastrographic Imaging, Medical Imaging Systems, and Computer Aided Diagnosis etc. Prof. Dr. Hossain has published about 50 research papers in different journals and conference proceedings. He was awarded Best Paper Awards in two international conferences. $\mathrm{He}$ is technical committee member of different conferences and journals. Prof. Dr. Hossain is a life fellow of Institution of Engineers, Bangladesh (IEB) and member of Institute of Electrical and Electronics Engineers (IEEE).

How to cite this paper: Rupak Bhakta, A. B. M. Aowlad Hossain, " Lung Tumor Segmentation and Staging from CT Images Using Fast and Robust Fuzzy C-Means Clustering", International Journal of Image, Graphics and Signal Processing(IJIGSP), Vol.12, No.1, pp. 38-45, 2020.DOI: 10.5815/ijigsp.2020.01.05 